\title{
Charm Production in Flux Tubes
}

\author{
C.E. Aguiar, ${ }^{1,2}$ T. Kodama,${ }^{1}$ R.A.M.S. Nazareth, ${ }^{1}$ and G. Pech ${ }^{3}$ \\ ${ }^{1}$ Instituto de Física, Universidade Federal do Rio de Janeiro, \\ C.P. 68528, 21945-970, Rio de Janeiro, Brazil. \\ ${ }^{2}$ European Centre for Theoretical Studies in Nuclear Physics and Related Areas, \\ Villa Tambosi, I-38050, Trento, Italy. \\ ${ }^{3}$ Instituto de Física, Universidade do Estado do Rio de Janeiro, \\ 20550-013, Rio de Janeiro, Brazil.
}

(July 17, 1995)

\begin{abstract}
We argue that the non-perturbative Schwinger mechanism may play an important role in the hadronic production of charm. We present a flux tube model which assumes that the colliding hadrons become color charged because of gluon exchange, and that a single non-elementary flux tube is built up as they recede. The strong chromoelectric field inside this tube creates quark pairs (including charmed ones) and the ensuing color screening breaks the tube into excited hadronic clusters. On their turn these clusters, or 'fireballs', decay statistically into the final hadrons. The model is able to account for the soft production of charmed, strange and lighter hadrons within a unified framework.
\end{abstract}

24.85.+p, 12.38.Lg, 12.39.Pn, 13.85.Rm

Typeset using REVTEX 


\section{INTRODUCTION}

Charm production in hadronic and nuclear collisions is presently a subject of considerable interest, as charmed particles are expected to be copiously created in relativistic heavy ion reactions at BNL's Relativistic Heavy Ion Collider (RHIC) and CERN's Large Hadron Collider (LHC). On one side, high energy electrons and muons coming from semileptonic decays of these charmed particles will provide a significant 'background' which may obscure dilepton signatures of a quark-gluon plasma [1]. On the other side, charm production itself may be an interesting probe of the QCD plasma due to the mass scale of $c$ quarks. It has been suggested that the observation of enhanced charm production in heavy ion collisions could provide a way for measuring the temperature of a hot gluon plasma [2] or the thermalization time of the initial partonic system [3].

In order to study charm production in relativistic heavy ion reactions one needs to have a good understanding of the production mechanisms operating in nucleon-nucleon collisions. Only in this case one can make meaningful extrapolations from hadronic to nuclear collisions, and use them to identify new phenomena associated with dense matter formation. Charm production in hadronic collisions has been extensively investigated with perturbative QCD. Of particular interest for such studies are the recent calculations of heavy quark hadroproduction carried out to order $\alpha_{s}^{3}$ by various authors [ [ $]$. These nextto-leading-order calculations seem to describe very well several experimental features of bottom production, but when applied to charm they give a $c \bar{c}$ cross section which depends strongly upon the choice of the renormalization-factorization scale. This renders it difficult to assess from direct comparisons with data whether hard processes really represent the dominant source of charm in hadronic collisions. Furthermore, there are important features of the experimental data which are not reproduced by these perturbative calculations. Some examples are the small back-to-back $p_{T}$ correlations found in the charm-pair distribution [5], or the enhancement of leading charm production observed at large $x_{F}$ [6].

This leaves open the possibility that non-perturbative mechanisms have an important 
role in charm production. In fact, the relatively low mass of the $c$-quark places the hadronic production of charm on the border between perturbative and non-perturbative phenomena. Therefore, approaches from the non-perturbative side may prove instructive. Such approaches are usually in the form of phenomenological models. Although less rigorous, they provide useful physical images whose parameters, like the string constant or the vacuum pressure, should be object of more fundamental theories. However, the usual mechanisms for soft particle production do not give a good description of charm hadroproduction. The commonly used string models predict negligible charm yields, because the tension of a quark-antiquark flux tube is too small for it to break into a $c \bar{c}$ pair [8]. Statistical (hydrodynamical) models also do not predict any significant charm production, as typical fireball temperatures are much smaller than the charm mass.

It is then somewhat surprising to find out that by properly blending the string and statistical approaches one is able describe very well the hadroproduction of charmed particles. Such a hybrid approach to particle production (we call it the "firetube" model) was developed in Ref. [7] to study the rapidity and transverse momentum distributions of pions and nucleons in hadronic collisions. In the present paper we show how the firetube model can be extended to describe charm and strangeness production.

The general idea of the firetube model is as follows. We assume that the colliding hadrons exchange soft gluons when they pass through each other, and as a result acquire color charges. The receding hadrons then become connected by a flux tube which confines the strong chromoelectric field created by these charges. Quark pairs (including $c \bar{c}$ ) are created by the chromoelectric field via the Schwinger mechanism, and the resulting color screening breaks the flux tube into lumps of highly excited hadronic matter ("fireballs") which subsequently decay thermally into the observed hadrons. The firetube model shares many aspects with the usual models based on the fragmentation of a classical string, such as the Lund model [8]. However, there exist basic differences. First, because of the gluon exchange, in the firetube model the string tension can be much larger than that of an elementary string between a quark-antiquark pair. Second, the final hadrons come from the thermal decay of 
fireballs. These points bring some new aspects into the mechanism of hadron production. For example, in a common string fragmentation model, the hadrons are produced directly from the break up of a quark-antiquark string via the Schwinger mechanism. Therefore, in such a framework the so-called $K / \pi$ ratio is closely related to the string constant $\kappa$. In contrast to this, the mechanism for pion production in the firetube model is essentially the thermal decay of fireballs, and there is no contradiction here between the small $K / \pi$ ratio and a large hadron-hadron string tension. Furthermore, if the string constant is as small as the standard value $\kappa \simeq 1 \mathrm{GeV} / \mathrm{fm}$ of a $q \bar{q}$-string, the Schwinger mechanism does not produce any significant amount of charm, as we have already mentioned. However, the Schwinger pair-creation rate is very sensitive to the value of $\kappa$, and the larger string constants found in the firetube model drastically change the picture, providing a mechanism for abundant non-perturbative production of charm.

The aim of this paper is to investigate such mechanism. A brief description of the firetube model is given in Sec. II, and results for pion production are presented. In Sec. [II] we discuss charm production in the framework of this model. The total charm cross section is calculated for proton-proton collisions and the result compared to experimental data. The longitudinal and transverse momentum distributions of charmed mesons and baryons are also obtained and compared to measurements. In Sec. IV we investigate strangeness production and discuss $K$ and $\Lambda$ spectra. Finally, Sec. $\square$ is used for some further comments and conclusions.

\section{THE FIRETUBE MODEL}

When two hadrons collide, several sea partons (assumed to be gluons here) may be exchanged between them. As a result, these hadrons become colored objects linked by a flux tube. Let $S$ be the cross section of this tube, which should be of the order of the geometrical size of the colliding hadrons. Let also $Q$ be the color charge at the end points of

the flux tube, measured in units of the elementary color charge $q_{o}$ of a quark $\left(q_{o}=\sqrt{4 / 3} g_{s}\right.$, 
where $g_{s}$ is the QCD coupling constant). The chromoelectric field $E$ inside the tube can be calculated from Gauss' law to be $E=q_{o} Q / S$. Placed in such a field, a quark whose color charge points into the $E$ direction in color space (we omit $S U_{3}$ algebra indices for simplicity) will experience a force $q_{o} E=q_{o}^{2} Q / S$. Also, the string constant $\kappa$ is related to the field energy density by $\kappa=E^{2} S / 2$. From this we get

$$
\begin{gathered}
\kappa=\kappa_{o} Q^{2} \\
q_{o} E=2 \kappa_{o} Q
\end{gathered}
$$

where we have defined $\kappa_{o}=q_{o}^{2} /(2 S)$. We identify $\kappa_{o}$ as the string constant of an "elementary" $(Q=1)$ color triplet string. It is worthwhile to mention that the scaling relations we are using - $S \sim Q^{0}, E \sim Q^{1}, \kappa \sim Q^{2}$ - are very different from what one obtains with the MIT bag model. In this case the balance between the field energy density and the vacuum pressure leads to an increase of the flux tube cross section with the charge, $S \sim Q^{1}$, and the chromoelectric field and string constant scale as $E \sim Q^{0}, \kappa \sim Q^{1}$. However, lattice QCD calculations of flux tubes generated by sources in different representations of the color group do predict that the scaling of $S, E$ and $\kappa$ with $Q$ is the one we have used above [9].

The end-point charge $Q$ is not necessarily the same for every collision. It fluctuates because different numbers of gluons can be exchanged, and also because of the $S_{3}$ addition of color charges. Assuming that each gluon exchange is a step of a random walk in color space [10], it can be shown that the charges generated on the hadrons after the exchange of $n$ gluons distribute sharply around the mean value $(3 / 2) \sqrt{n}$, where the $3 / 2$ factor accounts for the gluonic octet charge. Thus, $\kappa$ and $q_{o} E$ fluctuate around

$$
\kappa \simeq \frac{9}{4} \kappa_{o} n, \quad q_{o} E \simeq 3 \kappa_{o} \sqrt{n}
$$

showing that the exchange of even a modest number of gluons gives rise to string constants and chromoelectric fields significantly larger than the elementary ones. 
In order to determine completely the statistical distribution of the color charge $Q$ we must know the probability of having $n$ gluons exchanged in a collision. We assume that this is given by a truncated Poisson distribution,

$$
P_{n} \sim \nu^{n} / n !, \quad n \geq 1,
$$

where $\nu$ is a parameter related to the average number of gluons by $\bar{n}=\nu /\left(1-e^{-\nu}\right)$.

The constant chromoelectric field $E$ produces quark-antiquark pairs inside the flux tube by a process similar to the Schwinger mechanism of electron-positron creation in QED. The $q \bar{q}$ production rate per unit volume per unit time can be calculated (in the Abelian approximation) from Schwinger's formula

$$
\mathcal{R}_{q}=\frac{\left(q_{o} E\right)^{2}}{4 \pi^{3}} \sum_{n=1}^{\infty} \frac{1}{n^{2}} \exp \left\{-\frac{n \pi m_{q}^{2}}{q_{o} E}\right\}
$$

where $m_{q}$ is the quark mass. Corrections for the final state interaction of the $q \bar{q}$ pair can be introduced into this formula by taking $q_{o} E=2 \kappa_{o} Q-\kappa_{o}$. In our calculations we also corrected Eq.(50) for the finite transverse size of the flux tube, using the semiclassical formula of Ref. [11] (see their eq.27). For the cases of interest to us, the simple semiclassical result is not very different from the more precise correction of Ref. [12]. In principle we should also correct the Schwinger formula for the finite length of the flux tube [13. But it has been shown [11] that if the tube end-points are moving rapidly (which is our case) such corrections are suppressed by relativistic dilation effects. For this reason we have neglected longitudinal size corrections.

The quarks created by the chromoelectric field have their color charges aligned with $E$ in $S U_{3}$ space (the other orientations will be ignored as they have a much smaller production rate [10, [15]) and tend to screen the end-point charges $Q$, providing a mechanism for the flux tube fragmentation. Although other processes such as collective instabilities of the vacuum can be invoked to explain the break up of the tube into fireballs, we will assume that pair creation gives the dominant mechanism. This has the advantage of allowing for a simple estimate of the firetube fragmentation rate per unit length per unit time as 


$$
\omega \approx \frac{S}{Q} \sum_{q} \mathcal{R}_{q}
$$

where the sum extends over the quark flavors ( $u, d$ and $s$ in practice). This is, admittedly, a rough treatment of screening effects, but we did check that the typical break-up time we obtain in this way is comparable to the collapse time of the color field calculated with quark transport models [14].

The firetube model has a relatively small number of parameters. For proton-proton collisions we take $\bar{n}=2.0, \kappa_{o}=1 \mathrm{GeV} / \mathrm{fm}$ and $S=1.5 \mathrm{fm}^{2}$. Note that these values are quite reasonable: $\kappa_{o}$ is the usual $q \bar{q}$ string constant [8,15], and the firetube radius $R=\sqrt{S / \pi} \simeq$ $0.7 \mathrm{fm}$ is almost the same as that of the proton. A few other parameters define the minimum fireball mass $(1 \mathrm{GeV})$, regulate the behavior of the leading particles, and determine how the effective temperature and longitudinal expansion rate of a fireball depend upon its mass [7]. For the quark masses we use the constituent values $m_{u}=m_{d}=300 \mathrm{MeV}, m_{s}=450 \mathrm{MeV}$ and $m_{c}=1.5 \mathrm{GeV} 15$.

Having defined all this, we can perform Monte Carlo simulations of the firetube formation, its fragmentation into fireballs, and the thermal decay of these into the observed hadrons [7]. A typical result of such calculations is presented in Fig.1, where we show the rapidity distribution of charged particles (mostly pions) produced in proton-proton collisions at $\sqrt{s}=$ $20 \mathrm{GeV}$ and $53 \mathrm{GeV}$. We see that the model calculation is in good agreement with the experimental data [16] at both energies. Results of similar quality are obtained for the transverse momentum distributions.

\section{CHARM PRODUCTION IN THE FIRETUBE MODEL}

As it can be seen from Schwinger's formula, Eq.(15), the production rate of charmed quarks is extremely sensitive to the value of $q_{o} E$. For example, if $q_{o} E$ were of the order of $1 \mathrm{GeV} / \mathrm{fm}$ as usually quoted in the string model, the dominant exponential factor in Eq.(5) would be of the order of $10^{-15}$, and no reasonable interaction volume and time scale

for hadron-hadron collision would account for the observed charmed particle production 
cross section. On the other hand, in our model the average value of $q_{o} E$ is approximately $3 \mathrm{GeV} / \mathrm{fm}$ and the average value of the dominant exponential factor for charm becomes as large as $10^{-4}$, for the parameter values given above.

In order to obtain the charm production cross section we proceed as follows. First, we calculate the available space-time volume $V T$ as the total area swept by the flux tube in the space-time plane times the tube cross section $S$. This is completely determined if the fragmentation scheme of a firetube is specified. The total number of charmed quarks is then twice the value $V T \times \mathcal{R}_{c}$. In Fig.2 we show the total charm production cross section calculated in this way, and compare it to experimental data 17 20. The agreement is seen to be good, specially if we have in mind that the same parameter set is used at all energies. In our model, the energy dependence of the charm production rate comes essentially from that of the space-time volume $V T$ swept by the firetube, which increases asymptotically as $\ln (s)$

To calculate the momentum distribution of charmed particles, we assume that the $c$ quarks produced by the mean color field are distributed among the tube fragments with probabilities which are proportional to the mass of each fireball. The charmed particles are then emitted from the fireballs following their longitudinal expansion and thermal decay, in the same way as the other mesons [6]. In Fig.3 we show our calculation for the $x_{F}$ distribution of $D$ mesons produced in proton-proton collisions at $\sqrt{s}=27 \mathrm{GeV}$ (solid line) together with experimental points [17]. The agreement is very satisfactory. The transverse momentum distribution of charmed mesons predicted by the model is also in a good agreement with experimental data [17], as shown in Fig.4.

In addition to the particles coming from fireballs, hadronic spectra also get a contribution from the leading particles. The mechanism for tube breaking into fireballs assumes a fragmentation rate $\omega$ homogeneous in space-time. There is no apriori reason for this to remain valid at the two end-points which contain the valence quarks, because of the different boundary conditions. In fact, to reproduce the observed leading nucleon spectra we have to require that the two proton-like extremities detach from the firetube with a probability rate 
that is constant on their world-lines. When these hadrons separate from the firetube, there exists a chance that they turn into charmed particles, as the detaching mechanism should also be related to the production of $q \bar{q}$ pairs. Thus, the probability of having a charmed leading particle can be estimated as $\mathcal{R}_{c} / \sum_{q} \mathcal{R}_{q}$. We have calculated in this way the $x_{F}$ spectrum of leading charmed baryons $\left(\Lambda_{c}\right.$ 's) produced in proton-proton collisions, and the result is indicated by the dotted line in Fig.3. Note that these calculations do predict a substantial

leading particle effect, as the $\Lambda_{c}$ spectrum is much harder than the $D / \bar{D}$ distribution. For pion-nucleon reactions, where detailed observations of the leading charm effect have been performed [6], our calculations reproduce very well the experimental results [21]. We should mention that other non-perturbative effects, like intrinsic charm [22] or valons [23], have also been invoked to explain the leading particle behavior.

Another important feature of the firetube model is that we expect no strong transverse momentum correlations in the $D-\bar{D}$ pair spectra, as the charmed particles are emitted from fireballs which decay statistically. This seems to be consistent with most of the available data, which show almost no back-to-back angular correlations in the transverse plane [5].

A final point concerns charm production in hadron-nucleus collisions. Some preliminary extensions of our model indicate that the forward $\left(x_{F}>0\right)$ charm production cross section is essentially proportional to the target mass number $A$. However, for negative $x_{F}$ the charm production rate seems to increases more rapidly than linear in $A$.

\section{STRANGENESS PRODUCTION}

We have already seen that the large string tension arising in hadronic collisions can accommodate both the pion and charmed meson spectra quite satisfactorily. At this point one may worry about strangeness production. For simplicity we will assume here that strange particles are created solely through the Schwinger mechanism, in the same way as charmed particles. This means we are ignoring thermal production of strangeness, which in principle could take place inside the fireballs (for charm this process is certainly negligible 
because of the large mass of the $c$-quark). In Fig.5 we show the $x_{F}$ spectrum of neutral kaons produced in proton-proton collisions, calculated exactly as we did for the $D$ mesons. We see that the agreement of our results (shown as a solid line) with the experimental data [24] is very reasonable. Transverse momentum distributions predicted by the model are also in good agreement with experimental results. The production of strange leading particles can also be calculated along the same lines followed for the charmed ones. Our result for the spectrum of leading $\Lambda$ baryons produced in p-p reactions is shown in Fig.5 (dashed line). The accordance with experimental data [24] is again quite good.

\section{FINAL REMARKS}

In this paper we have discussed the possibility that a non-elementary flux tube is created between two colliding hadrons because of gluon exchange. This picture is consistent with charmed particles being produced at large rates by the color field inside the tube, via the Schwinger mechanism. We developed a simple model based on this idea and showed that it could account quantitatively for the production of charm in proton-proton reactions. The same model also describes very well the creation of lighter hadrons such as pions and kaons. It is a noteworthy aspect of our results that a successful description of the $p_{T}$ and $x_{F}$ spectra of such different particles as $\pi$ 's, $K$ 's and $D$ 's can be achieved in a unified manner.

An important issue refers to the competition between the mechanism for non-perturbative charm production proposed here and perturbative partonic processes. Hard processes certainly contribute to the charm yield but, as mentioned in the introduction, the present uncertainties in perturbative calculations make it difficult to determine the size of such a contribution. Even though we have shown that non-perturbative production by the color mean field can easily account for the observed charm cross section, we do not claim here that this mechanism represents the only significant source of charmed particles. It should be clear that the precise values of our charm cross section also suffer from uncertainties coming

from parameter values. For example, by adopting a slightly larger value for the mass of 
the $c$-quark we would reduce our charm production cross section without changing any of the model predictions for non-charmed particles. The basic point we want to stress here is that, within a reasonable set of parameters, our model shows that an important part of the charmed particles produced in a hadronic collision may have a non-perturbative origin. So, from our point of view, soft and hard mechanisms may well be both necessary in order to account for the bulk of charm production. What is certain is that several experimental observations [5,6] cannot be described on purely perturbative grounds, and require a significant contribution of soft mechanisms in order to be understood. In this paper we presented a consistent non-perturbative framework that can describe not only these but most features of charm production. For the reasons discussed in the introduction, it is of interest to investigate how the mean field mechanism for charm production discussed here scales to heavy ion collisions. Work along these lines is in progress.

\section{ACKNOWLEDGMENTS}

This work has been supported partially by CNPq, FINEP and FAPESP. G. Pech and C.E. Aguiar acknowledge CNPq Fellowships at CBPF and ECT*. 


\section{REFERENCES}

[1] R. Vogt, B.V. Jacak, P.L. McGaughey, and P.V. Ruuskanen, Phys. Rev. D 493345 (1994).

[2] E. Shuryak, Phys. Rev. Lett. 68, 3270 (1992).

[3] B. Müller, and X.-N. Wang, Phys. Rev. Lett. 68, 2437 (1992).

[4] P. Nason, S. Dawson, and R.K. Ellis, Nucl. Phys. B 303, 607 (1988); Nucl. Phys. B 327, 49 (1988); G. Altarelli, M. Diemoz, G. Martinelli, and P. Nason, Nucl. Phys. B 308, 724 (1988); W. Beenakker, H. Kuijf, W.L. van Neerven, and J. Smith, Phys. Rev. D 40, 54 (1989); R. Meng, W.L. van Neerven, G. Schuler and J. Smith, Nucl. Phys. B 339, 325 (1990); W. Beenakker, W.L. van Neerven, R. Meng, G. Schuler, and J. Smith, Nucl. Phys. B 351, 507 (1991); E. Laenen, J. Smith, and W.L. van Neerven, Nucl. Phys. B 369, 543 (1992); M.L. Mangano, P. Nason, and G. Ridolfi, Nucl. Phys. B 373, 295 (1992); S. Frixione, M.L. Mangano, P. Nason, and G. Ridolfi, Nucl. Phys. B 431, 453 (1994); E. Berger, and R. Meng, Phys. Rev. D 49, 3248 (1994).

[5] K. Kodama et al., Phys. Lett. B 263, 579 (1991); S. Aoki et al., Prog. Theor. Phys. 87, 1315 (1992); S. Barlag et al., Phys. Lett. B 302, 112 (1993); M. Adamovich et al., preprint CERN/PPE 94-214 (1994).

[6] M. Adamovich et al., Phys. Lett. B 305, 402 (1993); G.A. Alves et al., Phys. Rev. Lett. 72, $812(1994)$

[7] R.A.M.S. Nazareth, N. Prado, and T. Kodama, Phys. Rev. D 40, 2861 (1989); R.A.M.S. Nazareth, T. Kodama, and D.A. Portes Jr., Phys. Rev. D 46, 2896 (1992).

[8] X. Artru, and G. Mennesier, Nucl. Phys. B 7093 (1974); B. Anderson, G. Gustafson, G. Ingelman, and T. Sjöstrand, Phys. Rep. 97, 33 (1983).

[9] N.A. Campbell, I.H. Jorysz, and C. Michael, Phys. Lett. 169 B 91 (1986); H.D. Trottier and R.M Woloshin, Phys. Rev. D 48, 2290 (1993). 
[10] T.S. Biro, H.B. Nielsen, and J. Knoll, Nucl. Phys. B 245, 449 (1984).

[11] C. Martin, and D. Vautherin, Phys. Rev. D 40, 1667 (1989).

[12] H.-P. Pavel, and D.M. Brink, Z.Phys. C 51, 119 (1991).

[13] C.-Y. Wong, R.-C. Wang, and J.-S. Wu, Phys. Rev. D 51, 3940 (1995); R.-C. Wang, and C.-Y. Wong, Phys. Rev. D 38, 348 (1988).

[14] L. Wilets, and R.D. Puff, Phys. Rev. D 51, 339 (1995).

[15] A. Casher, H. Neuberger, and S. Nussinov, Phys. Rev. D 20, 179 (1979).

[16] C. de Marzo et al., Phys. Rev. D 26, 1019 (1982); A. Breakstone et al., Phys. Lett. 132B, 458 (1983).

[17] M. Aguilar-Benitez et al., Phys. Lett. B 189, 476 (1987).

[18] R. Ammar et al., Phys. Rev. Lett. 61, 2185 (1988).

[19] O. Erriquez et al., Phys. Scr. 33, 202 (1986).

[20] K. Kodama et al., Phys. Lett. B 263, 573 (1991).

[21] C.E. Aguiar, T. Kodama, R.A. Nazareth, and G. Pech, in preparation.

[22] S.J. Brodsky, C. Peterson, and N. Sakay, Phys. Rev. D 23, 2745 (1981); R. Vogt, S. J. Brodsky, and P. Hoyer, Nucl. Phys. B 383, 643 (1992); R. Vogt, and S. Brodsky, preprint LBL-35380 (1994).

[23] R.W. Hwa, Phys. Rev. D 51, 85 (1995)

[24] K. Jaeger et al., Phys. Rev. D 11, 2405 (1975). 


\section{FIGURES}

FIG. 1. Rapidity distribution of charged particles produced in p-p collisions at $\sqrt{s}=20 \mathrm{GeV}$ and $53 \mathrm{GeV}$. Experimental data are from Ref. [16]. The curves represent the result of Monte-Carlo calculations with the firetube model.

FIG. 2. Total cross section for charm production in p-p collisions as a function of c.m. energy. The line is the result of the firetube model. Experimental points (solid squares) are from Refs. [17,18]. The open squares refer to data obtained with proton-nuclei reactions [19,20].

FIG. 3. Feynman $x_{F}$ distribution of charmed particles created in p-p reactions at $P_{\text {inc }}=$ $400 \mathrm{GeV} / \mathrm{c}$. The solid and dashed curves correspond to our calculations for $D / \bar{D}$ mesons and $\Lambda_{c}$ baryons, respectively. Data points for $D / \bar{D}$ are from Ref. [17].

FIG. 4. Transverse momentum distribution of charmed mesons for the same reaction as in Fig.3.

FIG. 5. Feynman $x_{F}$ distribution of strange particles produced in p-p collisions at $P_{\text {inc }}=$ $205 \mathrm{GeV} / \mathrm{c}$. The solid and dashed curves correspond to our calculations for the $K^{0} / \bar{K}^{0}$ mesons and $\Lambda$ baryons, respectively. Experimental data are from Ref. 24]. 


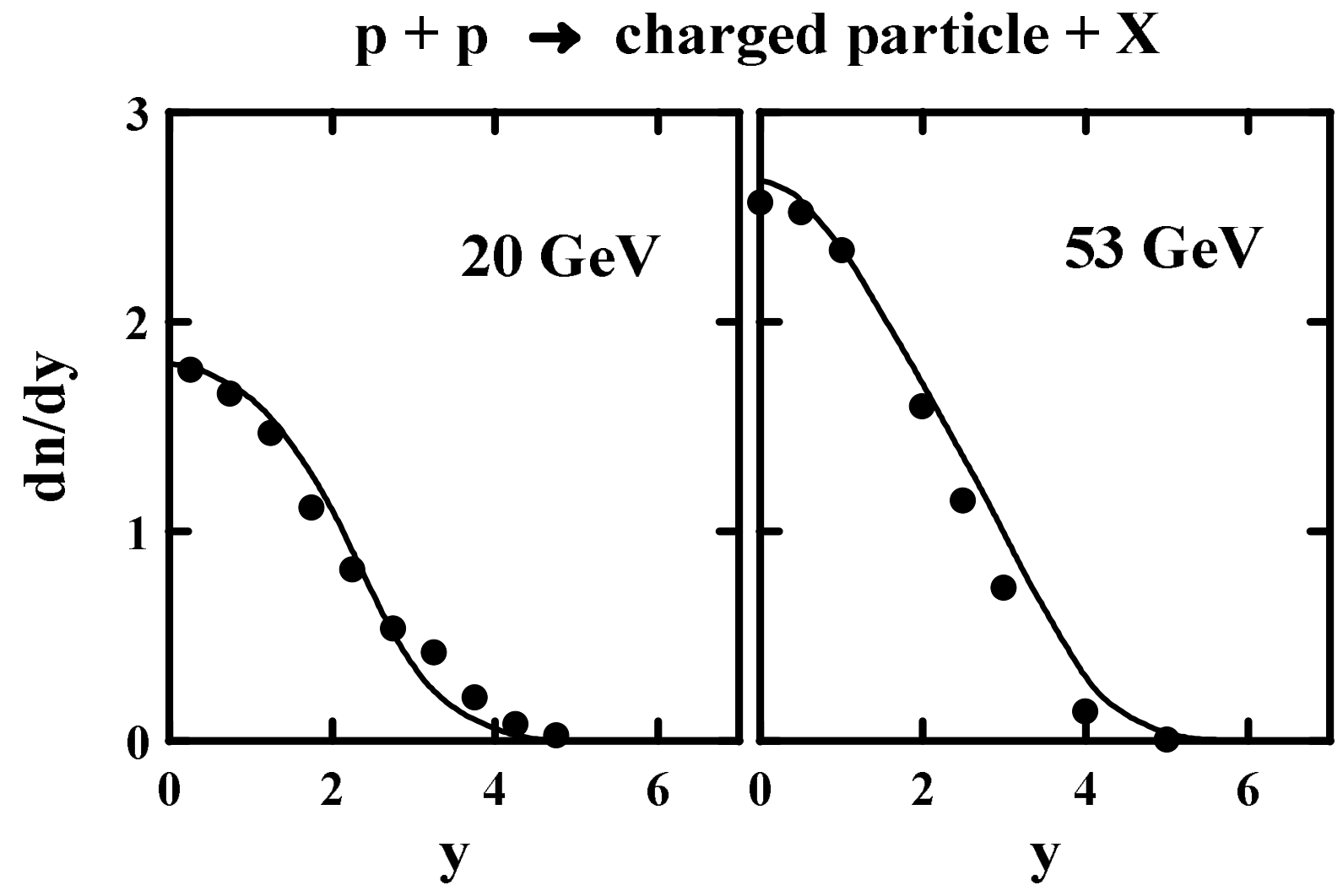

Fig.1 


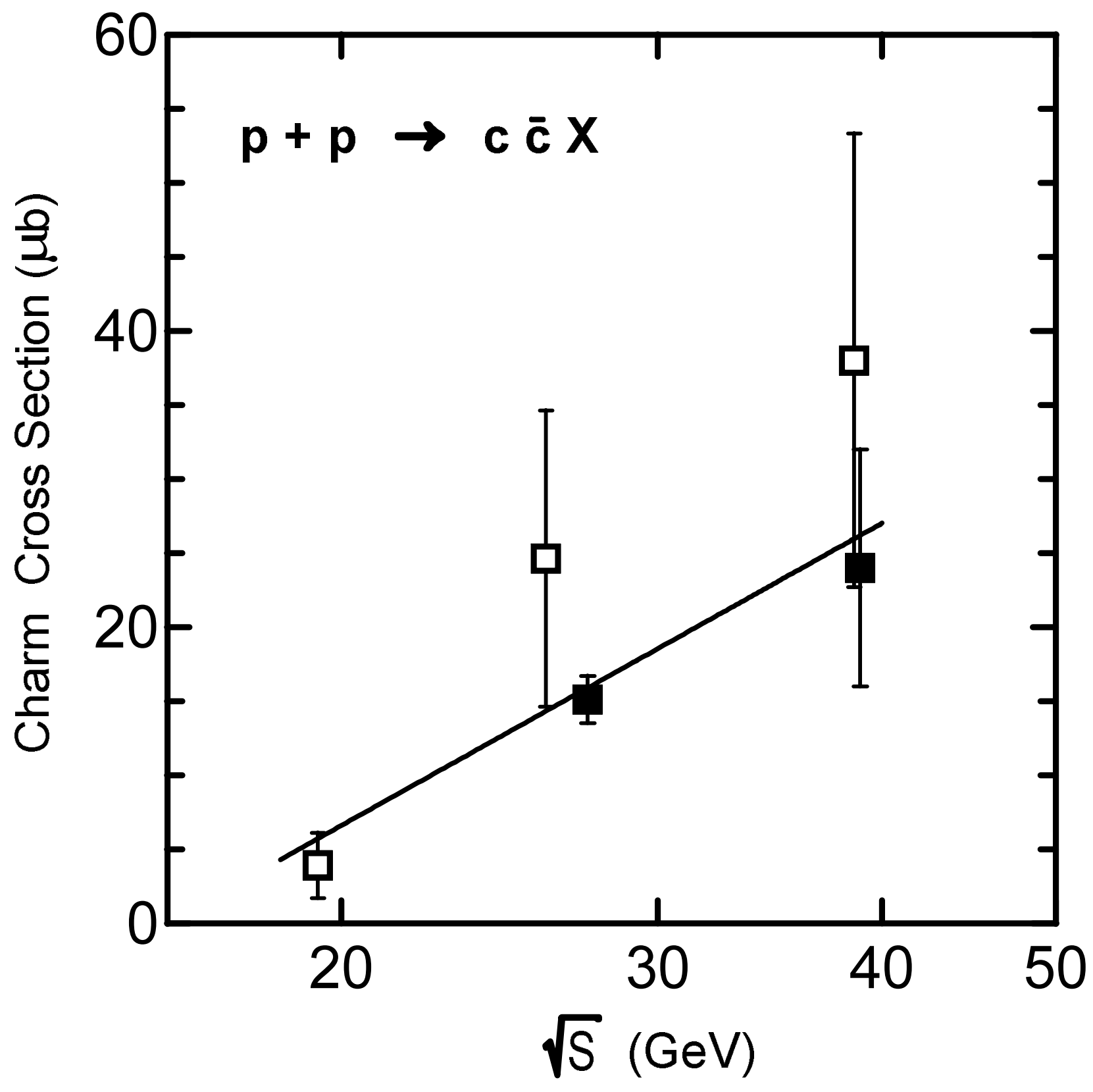

Fig.2 


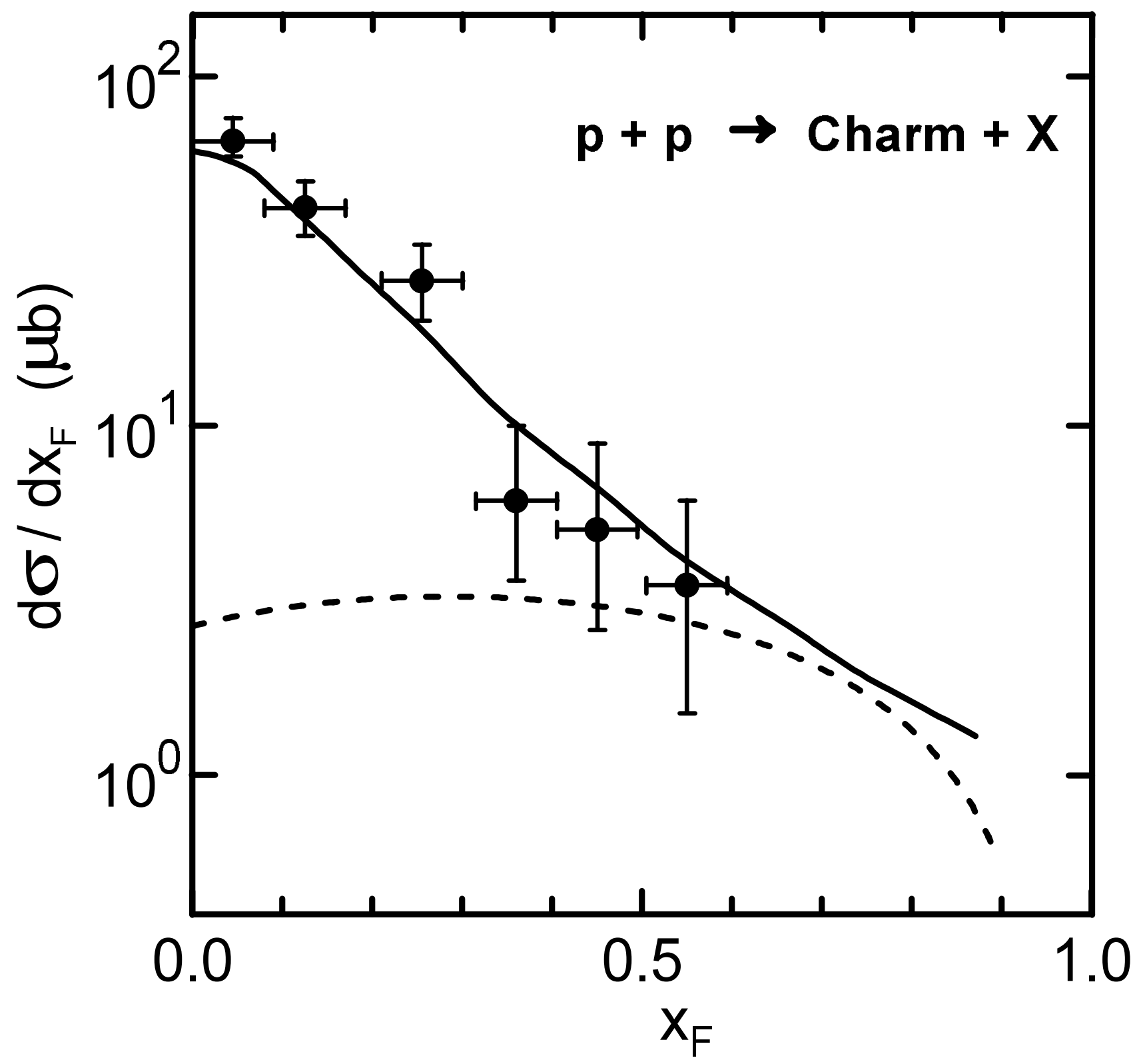

Fig.3 


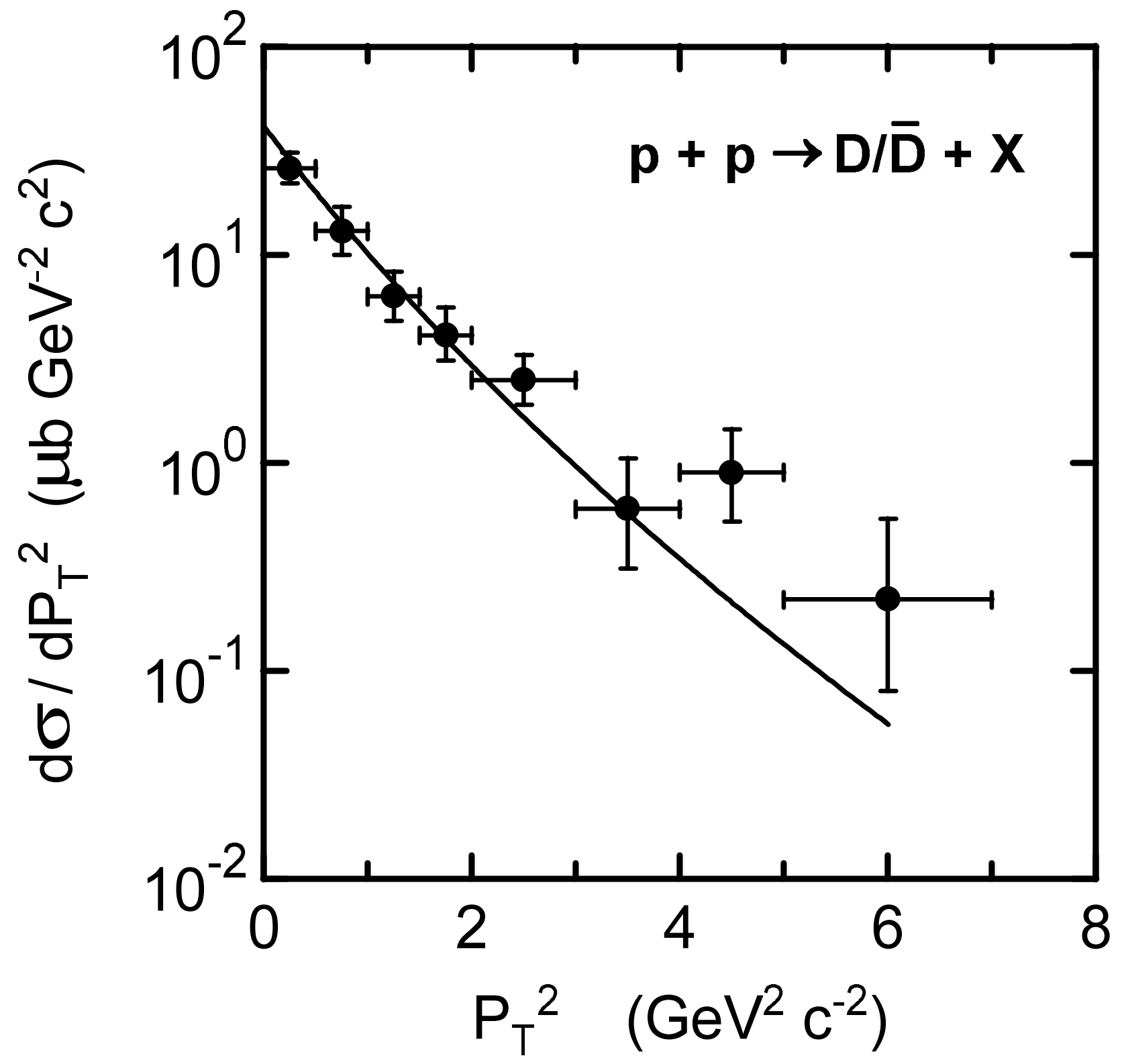

Fig.4 


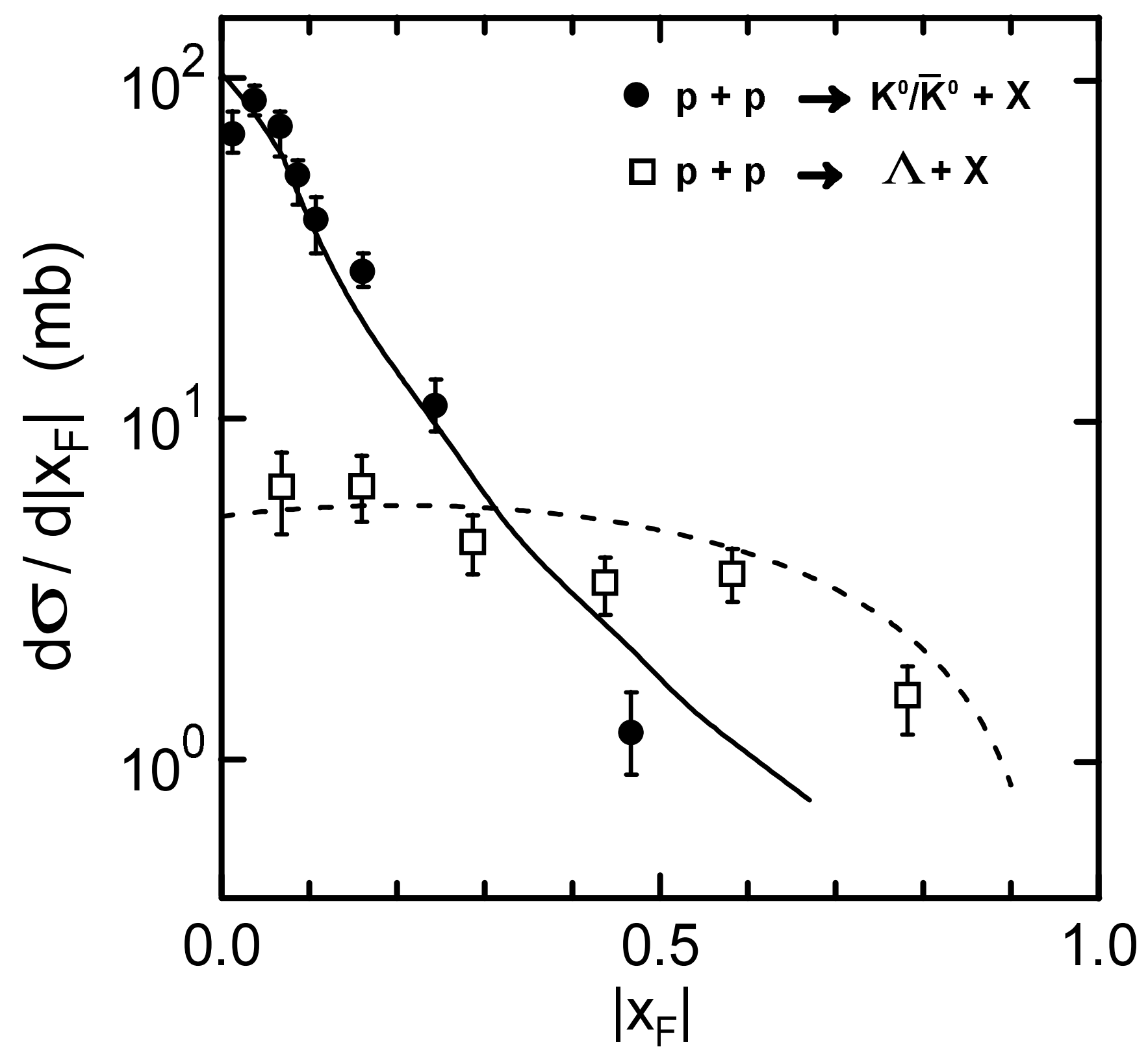

Fig.5 